\title{
Design on University Dormitory Charge Report Software Based on Crystal Report
}

\author{
Minghui Li \\ College of International Exchange, Bohai University, Jinzhou, P.R. China \\ liminghui0607@sina.cn
}

\begin{abstract}
Keywords: Crystal Report; university management; dormitory charge; report software; data accessing model; print output
\end{abstract}

\begin{abstract}
Crystal reports to achieve a high degree of integration with mainstream programming language, is currently the most widely used professional reporting system. The software is designed and developed based on crystal reports, provide diversified and standardized report to dormitory charges. Firstly, research reporting services architecture, and illustrate the major part; secondly, research data access patterns, including the pull model and push model, the two kinds; then, the data structure design, including the logical structure of table data and data types selection; Finally, study the report print output, give the output way of call report, and emphasizes the setting method of related properties. The development of this reporting system, solved the difficult problem of university dormitory management charge, save office costs, and improves the level of university information.
\end{abstract}

\section{Introduction}

The dormitory is student learning, life, friends, resting place, is the student's "second home", is an important position of quality education for students, but also an important window to show the school campus civilization. Dormitory management has become an important work of the school affairs; management is good or bad, directly related to the stability of higher education, which belongs to a very important part of the university teaching quality management assessment. The traditional manual management mode is low efficient in many aspects, such as records query, data statistics, graphs print, but also prone to error, unable to share information, cannot meet the needs of modern education information management. The use of advanced micro-computer, a mobile communication network and communication protocols, internetworking equipment and other information technology products, to integrate digital resources and information resource of school information centre, not only to strengthen the school dormitory management level, improve the comprehensive service quality, but also the need of the campus information construction. Under this background, develop the student dormitory management system, charge reporting software is an important component.

Crystal Reports is the industry's most professional, most powerful reporting system, in addition to powerful reporting capabilities, but also realized the integration and interface with the most popular development tools [1]. The software is based on crystal reports for development, provides diversified, standardized reporting for dormitory charges.

\section{Reporting Services Architecture}

In reporting services, a report server is implemented as a Windows service that consists of distinct feature areas that run in separate application domains. The service hosts report manager, the report server Web service, and background processing feature areas. Reporting services architecture is shown in Fig. 1.

For Fig.1 briefly described as follows:

(1) Service and Infrastructure. The Report Server Windows service is a consolidated set of applications that run in a single process. Configuration settings for the whole service are stored in RSeportServer.config, ReportServerServices.exe.config, and the report server database. 
(2) HTTP Listener. Reporting Services includes an HTTP listener that monitors incoming requests directed to HTTP.SYS on a specific port on the local computer. The host name and port are specified on a URL reservation when you configure the server.

(3) Authentication Layer. Reporting Services includes an authentication layer that verifies the identity of the user or application that makes the request. The following authentication types are supported: Windows integrated security, NTLM authentication, Basic authentication, Forms or custom authentication, and Anonymous access.

(4) Report Manager. Report Manager is a zero-footprint client that provides Web front-end access to the Report Server Web service. It is the out-of-the-box tool for viewing and managing report server content and operations.

(5) Report Server Web Service. The Report Server Web service is the core engine for all on-demand report and model processing requests that are initiated by a user or application in real-time, including most requests that are directed to and from Report Manager.

(6) Background Processing. Background processing refers to operations that run in the background and are initiated by the report server. Most background processing consists of scheduled report processing and subscription delivery, but it also includes report server database maintenance tasks.

\begin{tabular}{|c|c|c|c|}
\hline HTTP Listener & RPC \\
\hline $\begin{array}{c}\text { Front-end access to report } \\
\text { server items and operations }\end{array}$ \\
\hline UI Extensions
\end{tabular}

Fig. 1. Reporting services architecture

\section{Data Accessing Model}

Crystal reports connect to the database through the database driver. In order to provide flexible data accesses methods to developers, the crystal report database driver is designed to simultaneous provide data access Pull Model and Push Model [2].

\section{Pull model}

The so-called pull model, in accordance with the specified driver automatically linked to the database, and according to the pre-designed user query statement to "pull" data, pull" process is shown in Fig. 2 [3-5]. If the specific code is not compiled at run time, can use the pull model, according to the guide of report expert to operate.

Pull model applies to the database (or data file) does not require for data additional processing, the advantage is that the design is very convenient, can at any time preview the effect of the design; drawback is that when the application deployment is more complicated, need to set database link data sources and its driver. 


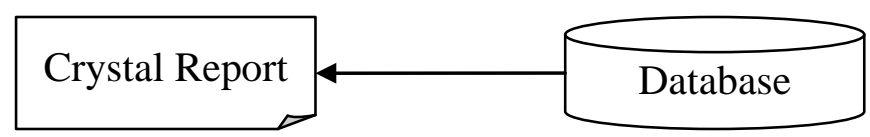

Fig. 2. Flow on "Pull model"

\section{Push model}

Push model requires developers to write their own code to link to a database, execute SQL statements to create the record set or data sets with the report field matches, and the resulting data is passed to the report, pushed the process is shown in Fig. 3 [6-7].

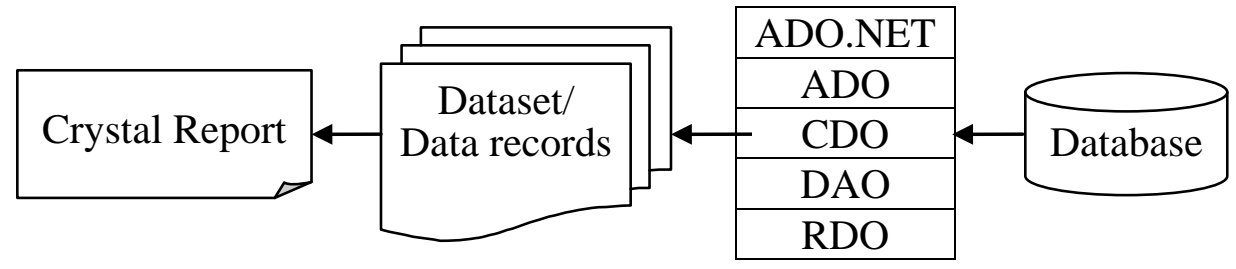

Fig. 3. Flow on "Push model"

Disadvantage of push model is complicated operation, needs to write more code, developers require a higher level. Advantage is reflected in two aspects: First, the developers have greater autonomy and control right for database, it can filter the data and then passed to the crystal report, can also be connected with other objects common database of program; the second, reporting engine does not directly contact to the database, but the use of the program created data set or data records set, and less affected by the engine itself in functional limitations, can through a variety of different data sources to extract data records and submit to the crystal report to display or print.

\section{Data Structure Design}

At present, many universities dormitory information management system, financial management of the dormitory charges is a relatively independent management system. As long as dormitory information management system, it must include the student's daily charge and management work, including charge situation of institute, department, classes, and apartment or personal items. At the same time, at any time, after obtaining print arrears personnel list and related information, provide the convenience for urging students to pay the arrears. Many universities dormitory charge report, but the most basic should include the following three:

(1) Student individual payment report, comprehensively reflect the detailed situation of each student payment. Report contents include "student number, the student's name, payment date, payments project, the amount payable, and the amount actually paid, the balance after the payment," and so on. The "balance after the payment" can be positive or negative, positive show payment is more, negative expressed as arrears. In the report, a "payment project" of a student holds a record.

(2) Sub-payment dormitory student payment statistics report, comprehensively reflect the detailed situation of each dormitory student charge paid. Report contents include "dormitory number, dormitory charge, electric charge, consumption, health costs, network charge, telephone fees, accommodation deposit," and so on. In the report, each dormitory representing a record, indicating the payment situation of dormitory in a period of time.

(3) Dormitory charge comprehensive statistical report, the whole school dormitory charges detailed statistics. Display information according to the institute, each institute accounted for a record. Report contents include "institute name, institute code, payment project, the amount payable, the amount actually paid, the balance after the payment, the payment rate," and so on.

\section{Report Print Output}

The PrintToPrinter method of the ReportDocument object will report sent to the computer's default printer. Call format is: 
myRD.PrintToPrinter(parameter 1, parameter 2, parameter 3, parameter 4)

Among them, the parameter 1 is the number of copies, integer; parameter 2 indicates whether automatic pagination, Boolean; parameters 3 represents a page where to start printing, integer; parameter 4 is printed to which pages, integer. If print the entire report, parameter 3 and parameter 4 are set to 0 .

If need more control over the printing operation, requires the use of PrintOptions class, the main attributes of the class as follows:

(1) PageContentHeight. Get the height of the page content.

(2) PageContentWidth. Get the width of the page content.

(3) PageMargins. Gets or sets the page boundary of the report. ApplyPageMargins method must be used to change, the members are as follow: bottomMargin, lower boundary value; leftMargin, left boundary value; rightMargin, right boundary value; topMargin, the boundary value.

(4) PaperOrientation. Gets or sets the current printer paper direction.

(5) PageSize. Gets or sets the size of the current printer paper. DefaultPageSize indicates the default settings for the printer.

(6) PaperSource. Gets or sets the current printer paper source. Can use an enumeration type to set, the members are as follow: Auto, Cassette, Envelope, EnvManual, FormSource, LargeCapacity, LargeFmt, Lower, Manual, Middle, SmallFmt, Tractor, Upper and so on.

(7) PrinterDuplex. Gets or sets the current double side printing options.

(8) PrinterName. Gets or sets the report used the name of the printer. An empty string indicates the default printer.

\section{Conclusion}

Report as an important form of information presentation, is an important part of application software systems, convenient, fast and accurately generate a variety of statistical reports, is an important work of software development. By developing university dormitory management system, dormitory managers can quickly and easily manage student dormitories, improve work efficiency, saving manpower, financial and material resources, to ensure data security, reliability, accuracy, while the dormitory management work standardization, procedures and systematic to avoid subjective and arbitrary. The development of this reporting system, to solve the problem of charge difficult, saving office costs, but also can improve the efficiency of the digital office.

\section{References}

[1] L. M. Zhang. With the example of learning Crystal Report. Beijing: Electronic Industry Press , 2004.

[2] J. Fu. W. Z. Zhao, X. Z. Mi, Y. S. Huang. Application of Crystal Reports under the NET platform. China Water Transport, 7(6), pp. 168-169, 2006.

[3] C. F. Xia, Y. Q. Yang. Research on Automatic Generation Technology of General Crystal Report. Computer Technology and Development, 23(5), pp. 54-57, 2013.

[4] H. G. Chang. Research on shipyard reporting system under Network Environment. Master's Degree of Harbin Engineering University, 2007.

[5] H. G. Zheng. Crystal report data transfer mode in .NET. Computer Programming Skills \& Maintenance, 14(11), pp. 44-46, 2007.

[6] C. F. Xia, Y. Q. Yang. Research on Key Technology of General Crystal Report Platform. Computer Technology and Development, 23(6), pp. 219-222, 2013.

[7] H. Zhang, S. L. Zhang, C. R. Zhu. Zheng. The application of crystal report to MIS of college students based on. NET. Journal of Changchun Institute of Technology Natural Science Edition, 15(2), pp. 117-121, 2014. 